\title{
La place du lecteur dans les fictions épistolaires orientales (1686-1735)
}

\section{Hanna Nohe}

\section{(2) OpenEdition}

1 Journals

Édition électronique

URL : http://journals.openedition.org/studifrancesi/16126

DOI : 10.4000/studifrancesi. 16126

ISSN : 2421-5856

Éditeur

Rosenberg \& Sellier

\section{Édition imprimée}

Date de publication : 1 juillet 2019

Pagination : 113-125

ISSN : 0039-2944

\section{Référence électronique}

Hanna Nohe, «La place du lecteur dans les fictions épistolaires orientales (1686-1735) », Studi

Francesi [En ligne], 187 (LXIII | I) | 2019, mis en ligne le 01 avril 2020, consulté le 25 janvier 2021. URL http://journals.openedition.org/studifrancesi/16126; DOI : https://doi.org/10.4000/studifrancesi. 16126

\section{(c) (i) (9)}

Studi Francesi è distribuita con Licenza Creative Commons Attribuzione - Non commerciale - Non opere derivate 4.0 Internazionale. 


\title{
DISCUSSIONI E COMUNICAZIONI
}

\section{La place du lecteur dans les fictions épistolaires orientales (1686-1735)}

\begin{abstract}
In his «Some Reflections on the Persian Letters» Montesquieu reflects on the success of his work, but also on other examples of "this type of novels" and supposes that it is popular "because the writers are describing their own feelings at the actual moment, which means that emotions are conveyed more powerfully" (transl. Margaret Mauldon). The present paper starts from these considerations in order to analyse such possibilities of reader participation in the genre Montesquieu refers to: oriental epistolary fictions of the early Enlightenment. Thus, the paper examines, next to Montequieu's Lettres persanes (1721), two further strongly influential texts of the genre: Gian Paolo Marana's L'Espion du Grand-Seigneur (1686) and the marquis d'Argens' Lettres chinoises (1735). The three aspects which characterize the genre will be analysed subsequently: first, the epistolary particularities, such as the effect of the real, temporal and affective immediacy and multiperspectivity; secondly, suspense created by the character's journey; and thirdly, the Oriental's character, which is not only 'other', but equally profoundly human, thus provoking the reader's empathy and favouring a change of perspective.
\end{abstract}

\section{Introduction}

Rien n'a plu davantage dans les lettres persanes, que d'y trouver, sans y penser, une espèce de roman. [...] D'ailleurs, ces sortes de romans réussissent ordinairement, parce que l'on rend compte soi-même de sa situation actuelle; ce qui fait plus sentir les passions, que tous les récits qu'on en pourrait faire. Et c'est une des causes du succès de quelques ouvrages charmants qui ont paru depuis les lettres persanes ${ }^{1}$.

Montesquieu commence ainsi les «Quelques réflexions sur les lettres persanes» qui paraissent dans le «Supplément» de la nouvelle édition de son œuvre en 1754. À travers l'expression «ces sortes de romans», il se réfère aux types de textes qui ressemblent aux Lettres persanes dans leur constellation narrative: un étranger d'une région lointaine se rend en France, en Angleterre, en Espagne ou dans le monde germanophone pour y observer les particularités politiques, religieuses et sociales. Au moyen de lettres destinées à ses compatriotes, il exprime ses observations. Nous désignerons ce genre de textes comme des «romans épistolaires de voyageurs orientaux fictifs»: bien que certains textes incluent également des voyageurs d'Amérique ${ }^{2}$, à propos du corpus analysé dans le présent article, comme nous le définirons ci-dessous, nous nous limiterons aux parties perçues comme orientales, ce qui nous per-

(1) Montesquieu, «Quelques réflexions sur les Lettres persanes». Pour toutes les citations tirées de Montesquieu, je m'appuierai sur l'édition approfondie de Jean Starobinski: MonTESQuIEu, Lettres Persanes, éd. présentée, établie et annotée par Jean Starobinski, Paris, Gallimard, 2003 [1973], «Folio classique».

(2) Cf. par exemple les Lettres d'une péruvienne (1747) de Françoise de Graffigny. 
mettra de déduire des conclusions plus concrètes. L'Orient sera entendu au sens large du terme, comprenant des régions du Proche, Moyen et Extrême-Orient, incluant ainsi autant l'Empire Ottoman, la Perse que la Chine. Cependant, soulignons que ces régions ne constituent que la provenance des personnages voyageurs et épistoliers fictifs, ceux-ci n'étant que le fruit de l'invention des auteurs, eux-mêmes français, anglophones, espagnols et germanophones.

Comme Montesquieu le signale lui-même dans la citation, les œuvres de ce genre se multiplièrent à la suite des Lettres persanes et suscitèrent l'enthousiasme du public. Il explique ledit succès par le fait que les romans épistolaires ne possèdent pas de narrateur; c'est l'épistolier lui-même qui relate ses expériences. De cette manière, chaque lettre est écrite par un narrateur autodiégétique à focalisation interne, pour emprunter les termes de Gérard Genette ${ }^{3}$. Montesquieu poursuit en affirmant que les passions sont ainsi mieux appréciées. Néanmoins, il laisse sous-entendu l'agent de l'appréciation: le lecteur. À travers son affirmation, il ne fait qu'allusion au récepteur extratextuel et à ce qu'il ressent tout au long de la lecture.

Les «Réflexions» de Montesquieu et leurs implications pour l'interprétation des Lettres persanes ont déjà été étudiées de manière détaillée, en particulier la signification de la formule de «chaîne secrète» ${ }^{4}$. Récemment, Jan Herman a présenté une nouvelle lecture qui est en partie liée à la question du lecteur ${ }^{5}$. Constatant des passages à la logique obscure dans les Lettres persanes, il détecte une chaîne secrète qui consiste en ces dichotomies. Ainsi, il arrive à la conclusion que ce «discours oblique» ${ }^{6}$ implique un «pacte de lecture» qui présuppose deux sortes de lecteurs: ceux qui entrevoient le discours caché et ceux qui ne le découvrent pas ${ }^{8}$. Herman part donc bien d'une lecture participative du lecteur, mais dans la lignée des structuralistes et, par conséquent, au niveau cognitif. Marie-Hélène Chabut a examiné la lecture dans les Lettres persanes à un niveau intratextuel et donc métafictionnel ${ }^{9}$. En repérant les «lieux du récit où le texte parle du livre ou de la lecture ${ }^{10}$, elle relève, d'une part, une critique de certains textes et constate, d'autre part, une réflexion de l'œuvre sur ellemême $^{11}$. Cette réflexion contient parfois des remarques auto-ironiques qui relativisent

(3) Cf. G. Genette, Figures III, Paris, Seuil, 1972, pp. 206 s. et 255 s.

(4) Ainsi, Pauline Kra isola certains blocs thématiques des Lettres persanes (P. KRA, The invisible chain in the "Lettres persanes", in «Studies on Voltaire and the Eighteenth Century» 23, 1963, pp. 7-60) et déclencha un véritable débat académique. Les articles poursuivent tous l'objectif de présenter un nouveau décodage de la «chaîne secrète»: J. Robert Loy (1961) l'interprète, avant l'écrit de Kra, comme la liberté, Richard L. Frautschi (1967) repère l'attitude d'étonnement comme un élément connectant, Nick Roddick (1974) trouve la chaîne dans les fragments érotiques et exotiques, Theodore Braun (1988) postule que le chaos est l'élément organisateur, Lucas A. Swaine (2001) soutient que la chaîne est un plaidoyer en faveur du parlementarisme et de la justice, alors que Randolph Paul (2005) défend la thèse que la structure fondamentale est l'écho qui existe entre les lettres dans l'ordre dans lequel elles sont présentées (cité selon R.P. Runyon, The Art of the Persian Letters. Unlocking Montesquieu's «Secret Chain», Newark, University of Delaware Press, 2005, pp. 14 ss. Toutes ces argumentations semblent plausibles; aucune n'est vérifiable comme la seule valide. Le mystère continue donc à émaner de la chaîne.

(5) J. Herman, Pacte de lecture et discours oblique, in Les "Lettres persanes" en leur temps, éd. Ph. Stewart, Paris, Classiques Garnier, 2013, «Rencontres» 62, pp. 137-156.

(6) Ibid., p. 155.

(7) Ibid.

(8) Cf. ibid., p. 153.

(9) M.-H. CHABut, Livre, lecture et dé-lire dans les "Lettres persanes", in L'épreuve du lecteur: Livres et lectures dans le roman d'Ancien Régime, Actes du VIII e colloque de la Société d'Analyse de la Topique Romanesque, Louvain-Anvers, 19-21 mai 1994, éd. J. Herman, P. Pelckmans, Louvain-Paris, Peeters, 1995, pp. 200-207.

(10) Ibid., p. 200.

(11) Cf. ibid., pp. 200 s. 
l'autorité du roman ${ }^{12}$. Ainsi, les allusions autoréférentielles engagent la réflexion du lecteur. Chabut, comme Herman, constate donc que les Lettres persanes provoquent l'activité cognitive du lecteur.

Cependant, les possibilités participatives particulières présentées au lecteur ${ }^{13}$ non seulement par les Lettres persanes, mais aussi par d'autres fictions épistolaires orientales, n'ont pas encore été évaluées de manière satisfaisante. Tel sera le sujet des pages suivantes: quelles sont les caractéristiques particulières du genre qui favorisent l'implication du récepteur? Quels effets s'ensuivent? Afin d'y répondre, nous nous appuierons, en plus des Lettres persanes, sur deux autres textes parus en langue française et influents dans l'évolution du genre: L'Espion du Grand-Seigneur (1686) de Gian Paolo Marana ${ }^{14}$ et les Lettres chinoises (1735) du marquis d'Argens ${ }^{15}$. Nous procéderons en trois temps qui correspondent aux différentes caractéristiques du genre. Nous commencerons par examiner les particularités liées à la forme épistolaire, mettant en évidence son effet de réel, l'immédiateté temporelle et affective et le multi-perspectivisme dû à la polyphonie. Ensuite, nous analyserons comment l'aspect du voyage permet de créer du suspense. Enfin, nous nous concentrerons sur les caractéristiques orientales et montrerons en outre que, à part créer ainsi une altérité, les caractéristiques profondément humaines des personnages sont enclines à provoquer l'empathie du lecteur et à favoriser son changement de perspective.

\section{Forme épistolaire: effet de réel, d'immédiateté et de multi-perspectivisme}

La lecture, dans le roman par lettres, a une double dimension. D'un côté, l'épistolier et son interlocuteur se situent à un niveau intratextuel, tandis que de l'autre côté, cette situation se répète entre l'auteur et le lecteur sur le plan extratextuel. En effet, la correspondance est mentionnée en tant que telle par les épistoliers à l'intérieur des lettres, créant ainsi un effet de réel qui brouille les limites entre la fiction et la réalité extrafictionnelle. Dans les Lettres chinoises de d'Argens en particulier, les personnages mentionnent souvent les écrits envoyés par d'autres ou l'absence de correspondance. Ainsi, dans la lettre 22, Choang, qui s'est rendu en Perse, explique à Yn-Che-Chan, demeuré à Pékin, les raisons de son long silence: «Quelques occupa-

(12) Cf. ibid., p. 202.

(13) Pour des raisons de simplicité linguistique, à partir de ce point nous emploierons la forme masculine. Toutefois, sémantiquement nous incluons également les lectrices, bien entendu.

(14) G.P. Marana, L'espion du Grand-Seigneur, et ses relations secretes: Envoyées à Constantinople. Contenant les evenements les plus considérables arrivés pendant la vie de Louis le Grand. Traduit de l'Arabe, Par le Sieur Jean-Paul Marana. Amsterdam, Henry Wetstein, 1688. Bien que le premier volume fût composé en italien, dès le second tome, le texte parut uniquement en français (cf. C.A. GIROTTO, «Marana, Gian Paolo», in Dizionario Biografico degli Italiani, éd. F.P. Casavola, Roma, Istituto della Enciclopedia Italiana, 2007, p. 410). Entre autres, Youmna Charara indique son influence non seulement au XVII , mais également tout au long du XVIII ${ }^{\mathrm{e}}$ siècle, ce qui est manifesté par le nombre important de titres commençant par «espion», tel que le montre aussi l'espion chinois de Goudar (cf. Y. CHARARA, La formation d'une série romanesque au XVIII siècle: les réécritures des "Lettres persanes", in Poétique de la pensée: Études sur l'âge classique et le siècle philosophique. En hommage à Jean Dagen, éd. B. Guion et al., Paris, Honoré Champion, 2006, pp. 201-218, ici p. 202).

(15) L'édition moderne et fondée de Jacques Marx servira de source aux citations: J.-B. DE BOYER, MARQUIS D'ARGENS, Lettres chinoises: ou correspondance philosophique, historique et critique, entre un chinois voyageur à Paris et ses correspondants à la Chine, en Moscovie, en Perse et au Japon, par l'auteur des 'Lettres juives' et des 'Lettres cabalistiques'. Édition établie et présentée par Jacques Marx, 2 vols., Paris, Champion, 2009, «L'âge des Lumières» 48. L'importance de cette œuvre de d'Argens au XVIII siècle peut être perçue dans des textes comme celui d'Oliver Goldsmith, The citizen of the world (1762), qui s'inspire de manière évidente de d'Argens, ce qu'indique également Marx dans son introduction (cf. ibid., p. 81). 
tions, mon cher Yn-Che-Chan, m'ont empêché de te donner de mes nouvelles, et si je ne t'ai pas écrit depuis quelque temps, n'en accuse point ma paresse. J'emploie toujours les moments que mes affaires me laissent, à m'instruire des mœurs et des usages des Persans ${ }^{16}$. Une telle évocation du contexte quotidien, dans lequel l'épistolier se trouve, invite le lecteur à s'imaginer plus concrètement la situation, si bien qu'elle apparaît plus authentique et donc plus proche. De la même façon, dans la lettre 98, c'est Sioeu-Tcheou, qui demande, depuis Dresde où il réside, des explications à YnChe-Chan concernant le fait que ce dernier ne lui a pas écrit: «Il y a longtemps, cher Yn-Che-Chan, que je n'ai reçu aucune des [sic] tes nouvelles; tire-moi, je te prie, d'inquiétude, et songe qu'un ami aussi tendre et aussi sincère que je suis, mérite bien que tu ne le laisses point dans une incertitude cruelle ${ }^{17}$. Ici, ce n'est pas l'épistolier, mais son interlocuteur qui n'a pas écrit. L'impératif «tire-moi [...] d'inquiétude» équivaut à l'ordre d'écrire de nouveau, ce qui réactive l'échange de lettres. Le lecteur extrafictionnel est également un récepteur de ces lettres, de manière qu'il se trouve au même plan de lecture que Sioeu-Tcheou: la lettre du personnage adressé est essentielle pour qu'il puisse poursuivre sa lecture.

Dans les Lettres persanes de Montesquieu, en revanche, ce n'est pas l'absence, mais la réception d'une lettre d'un tiers - Rhédi - ainsi que l'annonce de l'envoi d'une lettre d'un autre tiers - Rica - qu'Usbek transmet à Ibben dans la lettre 25:

J'ai reçu une lettre de ton neveu Rhédi: il me mande qu'il quitte Smyrne, dans le dessein de voir l'Italie; que l'unique but de son voyage est de s'instruire, et de se rendre par là plus digne de toi. Je te félicite d'avoir un neveu qui sera quelque jour la consolation de ta vieillesse. Rica t'écrit une longue lettre; il m'a dit qu'il te parlait beaucoup de ce pays-ci. La vivacité de son esprit fait qu'il saisit tout avec promptitude: pour moi, qui pense plus lentement, je ne suis en état de te rien dire ${ }^{18}$.

En évoquant d'autres personnages à l'intérieur de la missive, un réseau de communication s'établit, maintenu par les personnages en divers lieux: Ibben à Smyrne est informé de la lettre que son neveu a envoyé depuis Smyrne ainsi que de celle qu'il devrait recevoir de Rica. Or, à part le lecteur intra-fictionnel, Ibben, le récepteur réel à l'extérieur de la fiction est également informé des nouvelles. Ainsi, il attendra les lettres annoncées, devenant lui-même récepteur des lettres.

Dans le même temps, le rapport épistolaire fait allusion à une relation humaine; il insinue une dimension au-delà des lettres, comme l'évoque Yn-Che-Chan dans la missive 84: «Tes lettres, cher Sioeu-Tcheou, que je lis avec un plaisir infini, me font désirer ardemment le moment où je pourrai t'embrasser et jouir de ta conversation. Combien ne me sera-t-elle pas instructive, combien de choses ne m'apprendra-t-elle pas $[\ldots]$ ? $\gg^{19}$. L'épistolier anticipe le moment où les deux personnages entreront de nouveau en contact direct, à l'oral, et non plus par l'intermédiaire de l'écrit. Ainsi, les écrits surpassent les limites textuelles et font allusion à un réel extratextuel, de manière que pour le récepteur de ce niveau, les personnages de la fiction s'approchent du lecteur.

Par rapport au temps de la narration, l'aspect temporel de l'écriture épistolaire est thématisé de manière explicite. Ainsi, dans L'Espion du Grand-Seigneur, Mahmut explique les inconvénients, mais surtout les avantages d'écrire rarement:

Tu n'as receu aucune de mes Lettres dans les derniers Pacquets que j'ay envoyez, \& j'ay trouvé plus à propos de te faire sçavoir tout d'un coup, bien qu'un peu, plus tard ce que je

(16) D’ARgENs, Lettres chinoises, 1. 22, p. 312.

(17) Ibid., 1. 98, p. 812.

(18) MontesquiEu, Lettres persanes, 1. 25, pp. $92 \mathrm{s.}$

(19) D'ARgENS, Lettres chinoises, 1. 84, p. 719. 
n'aurois pû t'écrire qu'à trois fois; \& tu seras mieux informé que les autres, à qui j'ay écrit sur les premieres connoissances que j'ay eües. Quand on veut bien avoir un peu de patience, le temps fait connoistre la vérité, \& on a des confirmations des nouvelles, qui font qu'on est plus assuré de donner de bons avis ${ }^{20}$.

En commençant par évoquer une ellipse de temps et de lettres, l'épistolier constate que cela permet d'envoyer une synthèse des faits, c'est-à-dire les résultats des événements antérieurs. Afin de prévenir une possible accusation de négligence, il emploie une captatio benevolentiae argumentant en faveur de l'interlocuteur: celui-ci sera mieux informé. En effet, cette stratégie inclut également le récepteur extratextuel, car la patience évoquée par le personnage fait allusion à celle du lecteur externe qui doit parfois attendre les réponses à ses questions.

Par ailleurs, le fait que le moment de l'histoire et celui de la narration, dans la terminologie de G. Genette ${ }^{21}$, peuvent coïncider représente une des particularités du roman à lettres ${ }^{22}$. Une telle immédiateté narrative est illustrée à travers L'espion $d u$ Grand-Seigneur, lorsque Mahmut écrit à l'eunuque Egry Boyrou, dans la lettre 44:

Dans le moment que je t'écris je reçoy la nouvelle certaine de ma chute. Si je ne perds pas cette fois-cy la vie dans Paris: je seray peut-être plus heureux, \& je trouveray le moyen de mieux servir nôtre grand Empereur, dont la clemence égale la grandeur \& qui est au dessus de toutes les puissances de l'Univers. Le Cardinal de Richelieu me vient d'envoyer ordre de l'aler trouver; je finis même cette lettre à la haste, qui sera peut-être la derniere que j'écriray, craignant fort d'avoir esté découvert \& trahi. Si ma peur est vaine je t'apprendray par une autre dépêche les évenemens les plus considerables \& les plus singuliers de la vie de Henry: cependant je suis tout resolu, \& disposé à souffrir le martire ${ }^{23}$.

L'épistolier déclare l'identité de l'instant de rédaction de la lettre («Dans le moment que je t'écris») et celui dans lequel le personnage vit les événements («je reçoy la nouvelle»), avant d'en anticiper les conséquences possibles ( $\mathrm{Si}$ je ne perds pas cette fois-cy la vie dans Paris»). C'est uniquement par la suite que les faits sont expliqués. Enfin, la simultanéité de l'écriture et de l'événement conduit à une interruption de la première («Le Cardinal de Richelieu me vient d'envoyer ordre de l'aler trouver; je finis même cette lettre à la haste»). De cette façon, la sensation d'immédiateté crée un suspense qui se transmet au lecteur. Cette particularité narrative est employée avec efficacité afin de susciter l'intérêt du lecteur. Elle sera reprise de manière détaillée dans la deuxième partie.

Cette simultanéité de la narration et de l'histoire peut également porter l'épistolier à décrire le même fait de manière répétitive: parfois il relate un seul événement central à des destinataires différents et donc à plusieurs reprises. Dans les fictions épistolaires, cet aspect crée un effet d'authenticité, souligne l'importance de l'événement narré et augmente ainsi l'intérêt du lecteur extratextuel. Ainsi, le même Mahmut, cité dans le paragraphe précédent, évoque à nouveau dans l'écrit 45, soit une

(20) Marana, L'espion du Grand-Seigneur, 1. XXXIV, p. 111.

(21) Cf. GenetTe, Figures III cit., p. 71 s.

(22) Cf. J. vON STACKELBERg, Der Briefroman und seine Epoche: Briefroman und Empfindsamkeit, «Romanistische Zeitschrift für Literaturgeschichte»1, 1977, pp. 293-309, ici p. 294. Voir également W. Vosskamp, Dialogische Vergegenwärtigung beim Schreiben und Lesen. Zur Poetik des Briefromans im 18. Jabrbundert, «Deutsche Vierteljahrsschrift für Literaturwissenschaft und Geistesgeschichte» 45, 1/1971, pp. 80-116, ici p. 107.

(23) Marana, L'espion du Grand-Seigneur, 1. XLIV, pp. 168 s. 
lettre plus tard, la situation difficile dans laquelle il se trouve. Cependant, cette fois-ci, il s'adresse à un autre interlocuteur, «l'invincible Vizir Azem»:

Ton humble esclave, Mahmut, a un pas delicat à faire avec ce Ministre François [le cardinal de Richelieu]. Il m'a prié de luy donner quelque memoire sur ce que je sçay en general [...]: Mais que sur tout il avoit assez d'opinion de moy pour me confier, que je l'obligerois sensiblement si je luy donnois des memoires fort exacts des forces de l'Empire Ottoman, de la maniere dont il est gouverné, \& par où on le pourroit attaquer pour en diminuer la puissance. Je luy ay répondu fort modestement, que mon mestier n'estant que de dire mon Breviaire, je me sentois incapable de donner aucune instruction à un si grand homme que luy, qui n'ignoroit rien. Il m'a repliqué en riant que je ferois seulement ce que je pourrois [...]; enfin je n'ay pû me separer d'un homme si pressant sans luy promettre de luy donner quelque memoire. Tu sauras en son temps comment je me seray tiré d'un si mauvais pas, \& jusqu'où le zelle que j'ay pour ma Religion \& pour mon Souverain m'auront conduit $[\ldots]^{24}$.

L'épistolier reprend la situation délicate, mais ajoute des informations, cette lettre ayant été écrite à la suite de la rencontre mentionnée entre le protagoniste et le cardinal de Richelieu. De plus, le lecteur de l'époque pourra reconnaître l'homme politique évoqué, celui-ci ayant bel et bien existé, même si à partir de 1684, année de la publication de L'espion du Grand-Seigneur, Richelieu n'était plus en vie, son décès étant survenu en 1642. Par une telle évocation de personnages historiques, l'effet du réel est augmenté. Or, la fin de la situation reste ouverte en raison du style temporellement immédiat. Comme il s'agit d'une mission avec un double enjeu - un espion reçoit l'ordre de son 'ennemi' d'entrer à son service pour agir comme espion à son profit - ce qui peut conduire à la mort, la tension est exacerbée. L'effet est, d'une part, la rétention ${ }^{25}$ de la solution, d'autre part, le renforcement de l'importance du cas. Ainsi se crée un sentiment d'impatience chez le lecteur qui le pousse à poursuivre le récit en espérant une solution prochaine.

Enfin, l'absence de narrateur, caractéristique de l'échange épistolaire, offre une possibilité supplémentaire d'impliquer le lecteur par rapport à la perspective. Puisque chaque lettre est narrée de manière autodiégétique avec une focalisation interne de l'épistolier respectif, une pluralité de correspondants implique une pluralité de points de vue, suscitant à chaque fois l'empathie et la sympathie du lecteur. Or, lorsque ces différentes perspectives se contredisent au niveau logique, le récepteur extratextuel est non seulement impliqué de manière émotionnelle, mais aussi de façon cognitive. Ainsi, dans les Lettres persanes, vers la fin du roman, lorsque le 'soulèvement' des femmes dans le harem commence à se profiler, Usbek reçoit des lettres de divers personnages du sérail qui contiennent parfois des informations discordantes. Suite à une lettre de Narsit, l'eunuque le plus ancien qui annonça la mort du Premier Eunuque, Solim transmet l'information suivante à Usbek: «Si je gardais plus longtemps le silence, je serais aussi coupable que tous ces criminels que tu as dans le sérail. [...] tes femmes ne gardent plus aucune retenue: depuis la mort du grand eunuque, il semble que tout leur soit permis [...]. Je suis agité d'une colère vengeresse contre tant de perfidies $[\ldots] \gg^{26}$. Le ton bouleversé de ce personnage s'oppose nettement à

(24) Ibid., 1. XLV, p. 174 s.

(25) Ce terme est employé par Teresa Hiergeist pour désigner un effet de suspense dans la littérature. T. Hiergeist, Erlesene Erlebnisse. Formen der Partizipation an narrativen Texten, Bielefeld, Transcript, 2014, p. 177.

(26) Montesquieu, Lettres persanes, 1. 151, pp. 331 s. 
celui d'auparavant, créant ainsi une incohérence qui activera le lecteur: lequel des deux personnages dit la 'vérité?

En effet, l'insinuation de la discordance s'accentue dans la lettre postérieure, lorsque c'est Narsit qui reprend la parole: «Heureux Usbek! Tu as des femmes fidèles, et des esclaves vigilants: je commande en des lieux où la vertu semble s'être choisi un asile» ${ }^{27}$. Narsit présente une situation diamétralement opposée à celle décrite par Solim. Le lecteur doit donc évaluer la crédibilité des deux personnages et devient cognitivement actif. Il se souviendra probablement que, dans la lettre 147, le Premier Eunuque constate déjà un léger désordre, de telle sorte que le récepteur extratextuel aura tendance à donner raison à Solim. Au niveau émotionnel, il éprouvera, qui plus est, de la sympathie pour Solim et de l'hostilité envers Narsit.

Cependant, quatre lettres plus tard, dans la missive 156, le nombre de voix augmente, car celle de Roxane, qui est une des femmes qui représentait l'objet des descriptions, s'y ajoute: «L'horreur, la nuit et l'épouvante règnent dans le sérail: un deuil affreux l'environne: un tigre y exerce à chaque instant toute sa rage» ${ }^{28}$. Avec le mot «tigre», elle se réfère métaphoriquement à Solim qui, dans la lettre 153 d'Usbek, reçut le pouvoir d'agir à sa place. De cette manière, le personnage qui apparaissait auparavant comme le plus sensé, ressemble, dans la nouvelle perspective, à une bête sauvage, dangereuse et imprévisible pour les femmes. Le récepteur est de nouveau désorienté, situation qui sera encore aiguisée dans la lettre 159. Cette foisci, c'est encore Solim qui écrit: «Je me plains, magnifique seigneur, et je te plains: [...] Roxane, la superbe Roxane, ô ciel! [...] Je l'ai surprise dans les bras d'un jeune homme $[\ldots]\rangle^{29}$. Roxane, le dernier des trois personnages qui paraissait le plus fiable, est donc démasquée et son hypocrisie révélée. À travers ces rebondissements liés à des changements comportementaux, chaque épistolier se révèle comme un narrateur incertain, de sorte que le lecteur est déçu à maintes reprises. De plus, les incongruités dues à la multiplicité des voix narratives susciteront probablement le doute chez le récepteur extratextuel: peut-être commencera-t-il à douter de sa propre capacité de jugement et à se remettre en question lui-même.

\section{Voyageurs: ellipses temporelles et autres manières de créer le suspense}

La sensation d'immédiateté temporelle provoquée par la situation de correspondance est due au voyage fictif et permet d'introduire des interruptions de l'action et de créer un suspense qui suscite l'intérêt du lecteur et l'engage à poursuivre la lecture afin de connaître la suite du récit. Comme le voyage joue un rôle particulièrement important dans les Lettres chinoises, cette œuvre offre de nombreux exemples qui illustrent la manière dont cette situation narrative favorise la création d'un suspense. Ainsi, d'une part, nous pouvons trouver des ellipses par rapport à un épistolier absent qui résultent des modalités du voyage. Dès la lettre 2, Sioeu-Tcheou mentionne trois autres personnages dont il désire recevoir un message: «J'attends incessamment des nouvelles de Tiao et de Choang; le premier doit être arrivé en Moscovie, et le second sera sans doute bientôt à Ispahan. Marque-moi si Kieou-Che est parti pour le Japon, et prie-le de m'écrire» ${ }^{30}$. A travers cette évocation, l'épistolier introduit les autres

(27) Ibid., 1. 152, pp. 332 s.

(28) Ibid., 1. 156, p. 336.

(29) Ibid., l. 159, pp. 338 s.

(30) D'ARgENS, Lettres chinoises, 1. 2, pp. $197 \mathrm{~s}$. 
personnages principaux de même que leurs lieux de séjour et produit également du suspense chez le lecteur extratextuel qui se demandera si ces personnages sont bien arrivés. En outre, sa curiosité se focalisera sur ce qu'ils auront à raconter sur leur voyage et sur leur arrivée.

Ensuite, dans la lettre 8, l'absence de lettres est renforcée par Sioeu-Tcheou qui demande une fois de plus des nouvelles de Choang à Yn-Che-Chan:

Je suis en peine de la santé de notre ami Choang. Je ne reçois aucune de ses nouvelles; apprends-moi, je te prie, s'il t'a écrit depuis qu'il est arrivé à Ispahan. Dès que j'aurai reçu des lettres de Tiao, je t'en instruirai. Je ne sais s'il sera aussi content de son voyage en Moscovie, que je le suis de celui que j'ai fait à Paris ${ }^{31}$.

Le motif de la préoccupation de Sioeu-Tcheou est justement l'absence de lettres écrites par Choang. Or, en exprimant sa préoccupation, l'impatience est transmise au lecteur extratextuel, qui attend également une nouvelle lettre du personnage mentionné. De cette manière apparaît une tension émotionnelle qui induit à la poursuite de la narration. Elle est liée au voyage: hormis Yn-Che-Chan, tous les épistoliers sont en mouvement, ce qui implique de l'insécurité et donc de la tension. À un niveau de dramaturgie, les nouvelles qu'un personnage demande à un autre sur les absents évoquent l'interconnexion de tous, ce qui produit un effet d'authenticité et augmente l'attente qui se transmet au lecteur. Ainsi le récepteur réel se retrouve au même plan temporel et émotionnel que les lecteurs à l'intérieur de la fiction.

D'autre part, la situation du déplacement est employée comme excuse pour retenir des informations et augmenter ainsi le suspense. Ainsi, toujours dans les Lettres chinoises de d'Argens, Sioeu-Tcheou dans sa première lettre de Paris ne livre pas encore toutes les informations désirées:

Enfin me voilà à portée, cher Yn-Che-Chan, de me servir de la langue française, dont la connaissance nous a coûté plusieurs années d'étude. Je vais faire usage des instructions de nos amis les Européens; bientôt je pourrai vérifier si ce qu'il [sic] nous racontaient des mœurs des Français, est conforme à la vérité, et si les livres que nous avons lus par le canal des missionnaires, sont dignes de foi.

Je suis arrivé à Paris depuis deux jours: [...] Il faudra que tu me donnes le temps de revenir de ma première surprise [...]. Ne la regarde [cette première lettre], cher Yn-Che-Chan, que comme les discours d'un homme ébloui et troublé par confusion des objets. Un Siamois qui arrive à Paris, peut être comparé à ces malades qui ne sauraient souffrir qu'avec peine le grand jour, et qui sont obligés de s'accoutumer peu à peu à la clarté de cet astre ${ }^{32}$.

L'épistolier résume autant sa situation linguistique que celle du voyage. La première n'est probablement pas nouvelle pour son ami destinataire, mais elle l'est certainement pour le lecteur réel, et sert donc avant tout à clarifier le contexte et le propos du voyage et par là-même des relations. La situation du voyage, par contre, établit le cadre à la fois temporel et narratif: l'arrivée récente sur le lieu de séjour est la raison pour laquelle l'épistolier ne donne pas encore d'observations détaillées des mœurs françaises; de cette manière se produit un effet d'authenticité. Or, dans le même temps, cette explication sert d'excuse narrative pour retenir certaines constatations et accroître ainsi la curiosité du récepteur extratextuel.

(31) Ibid., 1. 8, pp. 22 s.

(32) D'Argens, Lettres chinoises, 1. 1, pp. 188 ss. 


\section{Provenance orientale: changement de perspective grâce à l'empathie}

La provenance orientale des épistoliers est, de même que les personnages et comme on l'a mentionné dans l'introduction, un artifice des auteurs; par conséquent, les aspects évoquant l'Orient, qu'il soit Proche ou Lointain, sont également imaginaires, même si les écrivains se sont basés sur des sources liées à des séjours réels dans les régions en question ${ }^{33}$. Par rapport à l'implication du lecteur, la provenance orientale crée avant tout deux effets: d'une part, elle suscite, surtout suite à la publication successive des Mille et Une Nuits à partir de 1704, des associations de sensualité et d'images voluptueuses, particulièrement vis-à-vis de la Perse ${ }^{34}$. Montesquieu profite de cette renommée pour créer une toile de fond romanesque, souvent analysée, avec les femmes du harem d'Usbek et ses eunuques qui évoquent tant ces associations positives qu'ils construisent un contre-monde régi par la violence et la tyrannie ${ }^{35}$. Ainsi, l'insurrection des femmes d'Usbek, déjà mentionnée dans la première partie, provoque une réaction violente de la part d'Usbek qu'il transmettra, en raison de son absence, au Premier Eunuque dans son sérail en Perse: «Recevez, par cette lettre, un pouvoir sans bornes sur tout le sérail: commandez avec autant d'autorité que moi-même: que la crainte et la terreur marchent avec vous» ${ }^{36}$. Cinq lettres plus tard, lorsqu'il est informé de la mort du Premier Eunuque et des tumultes croissants, il renforce cette manière violente de procéder, lorsqu'il écrit à Solim: «Je te mets le fer à la main. Je te confie ce que j'ai à présent dans le monde de plus cher, qui est ma vengeance. Entre dans ce nouvel emploi: mais n'y porte ni cœur, ni pitié»> ${ }^{37}$. L'appel à une absence de sentiments tendres évoque une tyrannie absolue. De cette manière, le récepteur est impliqué avec des sentiments variés: d'un côté, la fascination de ce monde fantastique l'attire et éveille sa curiosité; de l'autre, le système politique décrit comme cruel est enclin à le repousser et le convaincre du bien-fondé d'autres modèles proposés.

D'autre part, les personnages provenant de cultures orientales perçues comme différentes mais tout de même développées, comme la Chine en particulier, observent la société française à partir de points de vues étrangers et donc différents de ceux des récepteurs. Ainsi, Sioeu-Tcheou, qui compare les mœurs françaises et chinoises dans la lettre 21, juge le comportement des hommes français vis-à-vis de leurs amantes:

Ce en quoi je trouve les Français plus heureux que les Chinois, c'est que sous le prétexte qu'il n'est permis à un homme que d'avoir une seule femme, ils ne logent point chez eux leurs

(33) De manière simplifiée, History of The Present State of the Ottoman Empire (1668) de Paul Rycaut servait de source fondamentale pour la compréhension et représentation de l'Empire Ottoman; Les six voyages de Jean Baptiste Tavernier qu'il a fait en Turquie, en Perse, et aux Indes (1676) ont influencé tant les idées sur l'Empire Ottoman comme la Perse; le Journal du voyage du chevalier Chardin en Perse et aux Indes Orientales par la Mer Noir et par la Colchide (1686) étaient un deuxième recours important pour l'imaginaire sur le Perse; enfin, la compilation de Jean-Baptiste Du Halde, Description [...] de la Chine (1736) fut étudiée intensivement par d'Argens, comme il indique lui-même dans sa préface à la quatrième partié des Lettres chinoises, p. 765.

Paul Vernière et Catherine Volpilhac-Auger, dans l'introduction à leur édition des Lettres persanes expliquent de manière détaillée l'emploi des sources par Montesquieu. Cf. MonTESQuIEU, Lettres persanes, Edition de Paul Vernière, mise à jour par Catherine Volpilhac-Auger, Paris, Librairie Générale Française, 2005 [1960], p. 29. Voire également l'analyse comparée dans H. NoHE, Fingierte Orientalen erschaffen Europa. Zur Konstruktion kultureller Identitäten im Reisebriefroman der Aufklärung, Paderborn, Wilhelm Fink, 2018, pp. 67 ss. et 176 ss.

(34) Cf. P. MarTino, L'Orient dans la littérature française au XVII et au XVIII siècle, Genève [Paris], Slatkine Reprints [Hachette], 1970 [1906], p. 253.

(35) Cf. M. Delon, Un monde d'eunuques, «Europe» 574, 1977, pp. 79-88.

(36) Montesquieu, Lettres persanes, 1. 148, p. 329.

(37) Ibid., 1. 153, p. 333. 
maîtresses et ne les voient uniquement que dans certains moments destinés aux plaisirs: ainsi ils sont moins accablés des soins auxquels expose un ménage nombreux et composé de plusieurs femmes qui doivent vivre ensemble ${ }^{38}$.

L'épistolier, issu d'une société dans laquelle il est officiellement légitime d'avoir plusieurs femmes, juge évidemment ce fait comme acceptable. Ce qui lui semble digne de discussion est plutôt la question du lieu du logement de celles-ci, dont il évalue la situation en France comme meilleure par rapport à celle en Chine. Cette position se distinguera probablement de celle du récepteur français de l'époque où le fait d'avoir des maîtresses n'est certainement pas officiel. Or, en percevant une telle position différente de la sienne, il commencera probablement à réfléchir à sa propre opinion. Car, comme le note Hiergeist, plus les évaluations sont diverses, plus le récepteur devient cognitivement actif ${ }^{39}$.

Néanmoins, en dépit des différences culturelles, les textes sont capables de susciter l'empathie et donc la disposition émotionnelle du lecteur à changer de perspective. Elle est facilitée par le fait que les épistoliers éprouvent des sentiments profondément humains qui les unissent au récepteur. En décrivant leurs expériences vécues durant le déplacement, les voyageurs expriment autant leurs joies que leurs peines. Ce sont surtout ces dernières qui suscitent de l'empathie. Ainsi, Mahmut décrit ses plaisirs, mais aussi ses déceptions en amour, sentiments humains universels. L'épistolier se sent attiré par le personnage de Darie, qui apparaît uniquement à travers les propos de Mahmut:

Il y a long-temps que la belle Grecque, dont tu me demandes des nouvelles avec tant d'empressement s'est retirée en france, \& il y a environ 80. Lunes qu'elle s'est mariée avec un grand negociant François [...]. Ce fut à la Cour \& en presence du Roy mesme que je vis la premiere fois Darie [...]: \& dans le même instant je sentis naistre dans mon ame un secret desir d'en obtenir.... [...] Depuis ce temps-là je l'ay toûjours aimée [...]. Darie est une jeune personne dont le cœur est noble, \& dont les manieres sont agreables $[\ldots]^{40}$.

En faisant allusion aux statues antiques, la description de cette femme telle une «belle Grecque» permet d'évoquer de manière détournée une sensation érotique. Cela se confirme par la suite lorsque le protagoniste exprime ouvertement ses sentiments amoureux («je l'ay toûjours aimée»). Cela éveillera la curiosité et l'empathie chez le lecteur. En revanche, l'information selon laquelle elle s'est mariée suscitera probablement des sensations de confusion et de frustration, puisque la poursuite de l'histoire d'amour semble compromise. Toutefois, la tension émotionnelle est maintenue par l'ajout suivant: «Elle me dit une fois; Mahmut, ayons une amitié tendre l'un pour l'autre; mais aimons la vertu plus que nostre amitié. Montre moy l'histoire, \& enseigne moy aussi la Geographie, afin que [...] je puisse sçavoir en combien de parties cette terre, qui paroist si belle, est divisée» ${ }^{41}$. De cette manière, le récepteur extratextuel est informé de la poursuite du contact, ce qui apaisera sa frustration, et il songera peut-être à la probabilité d'une histoire d'amour. Effectivement, la lettre 83, déjà citée dans le paragraphe antérieur, l'évoquera et mènera à un autre sentiment: le chagrin.

(38) D’ARgENs, Lettres chinoises, 1. 21, p. 306.

(39) Cf. T. HiERGEIST, Erlesene Erlebnisse cit., p. 243.

(40) Marana, L'espion du Grand-Seigneur, 1. 69, pp. 264 s.

(41) Ibid., pp. 267 s. 
Le personnage peut également provoquer l'empathie dans des situations étrangères au lecteur, mais celui-ci pourra les comprendre grâce au caractère universel des sentiments humains. Ainsi, dans L'espion du Grand-Seigneur, Mahmut éprouve de la nostalgie quant à son environnement d'origine:

Tu releves d'une grande maladie, \& j'en attens une. Il y a déja quelques jours que j'ay une langueur qui m'abat extremement, mais par la grace de l'immortel je ne suis pas encore en état de recourir au Medecin. La lettre que j'ay reçû [sic] de toy cette Lune-cy a apporté quelque soulagement à la tristesse qui m'accabloit, \& qui m'est un mal assez ordinaire, dans la necessité où je suis de vivre éloigné de mes amis, de ma patrie, \& mesme pour ainsi dire de ma Religion $[\ldots]^{42}$.

Ce que le personnage nomme d'abord une «maladie», se révèle par la suite comme une grande tristesse causée par l'éloignement de sa terre natale. Il décrit ce que nous désignerions aujourd'hui comme un sentiment dépressif résultant d'une immense nostalgie. Or, la tristesse est un sentiment universel que le récepteur autochtone connaît aussi bien que le personnage turc. Ainsi, le lecteur partage avec Mahmut une expérience qui les unit de manière émotionnelle, de telle sorte qu'il éprouvera non seulement de l'empathie, mais également de la commisération envers le personnage confronté à une situation émotionnelle difficile. Cette sensation est renouvelée chaque fois que le personnage revient sur sa tristesse, comme il le fait dans la lettre 66, lorsqu'il exprime son souhait de «pouvoir rendre des gens aussi malheureux que je suis» ${ }^{43}$. Lorsqu'il demande ensuite à son interlocuteur Dgnet Oglou «Ecris moy souvent» ${ }^{44}$, la cause de l'absence des amis est évoquée de nouveau. Quand, enfin,

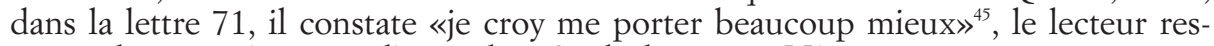
sentira la joie qu'éprouve l'épistolier. Sa déclaration «[j]'attens avec une impatience qui n'est pas imaginable, la saison qui couvre la Campagne de fleurs, qui fait croistre l'herbe des prez, qui donne aux arbres leurs feuilles ordinaires, \& qui ranime les petits oyseaux, pour esperer de recouvrer ma santé, n'y ayant que le printemps qui puisse me la redonner ${ }^{46}$ confirme une nouvelle fois sa mélancolie et suscite un sentiment de pitié chez le lecteur pour le personnage malheureux.

De manière semblable, dans les Lettres persanes, c'est Usbek qui, vers la fin du roman, exprime son mal du pays:

Heureux celui qui, connaissant tout le prix d'une vie douce et tranquille, repose son cœur au milieu de sa famille, et ne connaît d'autre terre que celle qui lui a donné le jour! Je vis dans un climat barbare, présent à tout ce qui m'importune, absent de tout ce qui m'intéresse. Une tristesse sombre me saisit; je tombe dans un accablement affreux: il me semble que je m'anéantis; et je ne me retrouve moi-même, que lorsqu'une sombre jalousie vient s'allumer, et enfanter dans mon âme la crainte, les soupçons, la haine et les regrets. [...] Malheureux que je suis! Je souhaite de revoir ma patrie $[\ldots .]^{47}$

La situation que le personnage décrit - la mélancolie causée par l'éloignement des personnes et des objets chers - s'oppose certainement à celle du lecteur autochtone, qui, en lisant ce passage, se trouve probablement chez lui, près de sa famille et

(42) Ibid., 1. 53, p. 219

(43) Ibid., 1. 66, p. 257.

(44) Ibid.

(45) Ibid., 1. 71, p. 276.

(46) Ibid., p. 280.

(47) Montesquieu, Lettres persanes, 1. 155, pp. 334 s. 
de ses amis. Malgré tout, le sentiment de manque et de tristesse lui sera familier, et il éprouvera de l'empathie pour une situation qui lui est étrangère.

Ce processus d'empathie et de changement de perspective va encore plus loin dans le cas du Premier Eunuque qui, toujours dans les Lettres persanes, décrit son état intérieur de la manière suivante:

[...] enfermé dans une affreuse prison, [je] suis toujours environné des mêmes objets, et dévoré des mêmes chagrins. Je gémis accablé sous le poids des soins et des inquiétudes de cinquante années; et, dans le cours d'une longue vie, je ne puis pas dire avoir eu un jour serein et un moment tranquille [...].

Voilà comme j'ai passé ma misérable jeunesse: je n'avois de confident que moi-même. Chargé d'ennuis et de chagrins, il me les falloit dévorer; et ces mêmes femmes que j'étois tenté de regarder avec des yeux si tendres, je ne les envisageois qu'avec des regards sévères: j'étois perdu si elles m'avoient pénétré: quel avantage n'en auroient-elles pas pris! [...]

Enfin les feux de la jeunesse ont passé; je suis vieux, et je me trouve, à cet égard, dans un état tranquille; je regarde les femmes avec indifférence, et je leur rends bien tous leurs mépris, et tous les tourments qu'elle m'ont fait souffrir ${ }^{48}$.

Le personnage de l'eunuque décrit sa situation, la monotonie de sa vie quotidienne, tant locale que sociale, ce qui invite à se l'imaginer, de même que les sentiments qui en résultent. Le récit de son histoire souligne cette perspective, car elle élargit l'uniformité quotidienne non seulement au présent, mais tout au long de la vie humaine. Par conséquent, son attitude agressive envers les femmes apparaît compréhensible. Le point de vue subjectif, interne, invite le lecteur à éprouver de l'empathie et à s'identifier au niveau émotionnel. Cela impliquera probablement un changement de perspective et, par la suite, de jugement. Ainsi, il est possible que le récepteur change d'opinion vis-à-vis de l'attitude de Fatmé dans la lettre VII où elle évoque les eunuques comme «ces eunuques affreux, dont la moindre imperfection est de n'être point hommes» ${ }^{49}$ et qu'il éprouve de la compassion envers l'eunuque qu'il verra désormais comme un être humain privé de sa nature virile et de son autodétermination. De cette manière, le lecteur est prêt à appréhender son propre monde à travers la perspective de l'autre.

\section{Conclusion}

La célèbre citation des «Réflexions» de Montesquieu nous a conduits à nous interroger sur les mécanismes à travers lesquels les fictions épistolaires orientales impliquent la participation active, qu'elle soit émotionnelle ou cognitive, du récepteur et produisent l'effet plaisant évoqué par Montesquieu. En analysant trois œuvres représentatives du genre, nous avons mis en évidence plusieurs éléments résultant de la particularité du genre d'être à la fois un texte épistolaire, un récit de voyage et une fiction orientale. Ainsi, l'échange de lettres est représenté de manière explicite et évoque ainsi un effet du réel pour le lecteur extrafictionnel qui reçoit lui-même les lettres. De plus, la simultanéité de la narration et de l'histoire, caractéristique du roman épistolaire, permet d'intégrer des interruptions de la narration et d'augmenter ainsi le suspense. Enfin, l'absence de narrateur permet de créer une polyphonie de différents personnages qui se contredisent mutuellement. Dans les Lettres persanes 
en particulier, une telle stratégie de multiplication des narrateurs non fiables active le récepteur cognitivement.

L'aspect du voyage propose des techniques pour créer un suspense. En raison des déplacements, certains personnages ne répondent pas aux écrits, ce qui provoque l'inquiétude des autres épistoliers et de l'impatience chez le lecteur avide de découvrir la suite. En outre, l'arrivée d'un voyageur à un nouvel endroit sert de prétexte pour retenir certaines informations et susciter la curiosité du récepteur davantage.

Enfin, la provenance orientale des épistoliers implique le lecteur à la fois de manière cognitive et émotionnelle. D'une part, les auteurs s'inspirent de l'imaginaire de l'Orient pour en créer un autre dont le récepteur peut se différencier quant à ses jugements, comme face à la brutalité dans le sérail des Lettres persanes ou le jugement de la polygamie dans les Lettres chinoises. D'autre part, le compte-rendu des émotions personnelles permet une identification immédiate à l'épistolier. Le fait qu'il s'agisse, dans la fiction, de personnages étrangers, est relégué au second plan. Dès lors, ce qui prévaut n'est pas la nationalité ou la culture du lecteur, mais l'aspect humain qui lie le récepteur au personnage. C'est donc à travers la description des sentiments d'une situation vécue de manière personnelle et directe que le lecteur parvient à s'identifier à l'épistolier oriental. En prenant parti, il se montre malgré tout prêt à changer de perspective. La flexibilité mentale est à la fois la prédisposition à relativiser les points de vue et à remettre en question des habitudes ou des manières de penser considérées comme immuables. Ainsi, l'implication émotionnelle est la condition d'une participation cognitive et évaluative qui influencera le récepteur non seulement dans la lecture des romans, mais aussi probablement dans la perception de sa propre réalité au-delà de la fiction. 